\title{
Radiographic Evaluation of the Bone Remodeling on Tilted Osseointegrated Implants: Follow-up at 1 Year
}

\author{
Maddalone Marcello ${ }^{1}$, Mirabelli Luca르, Breschigliaro Sara ${ }^{3}$, Baldoni Marco ${ }^{4}$
}

\begin{abstract}
Aim: The purpose of this study was to evaluate the degree of vertical peri-implant resorption around implants inserted with an inclination increased more than $10^{\circ}$ at a distance of at least 1 year from insertion.

Materials and methods: For the realization of the study, a sample consisting of 47 patients for a total of 115 implants was selected. We excluded all those subjects whose conditions could have compromised the outcome of the treatment. An orthopantomography of the dental arches was made using Orthophos XG 3D Sirona at time T0 (at the end of definitive insertion of prosthesis) and at time T1 (at least 1 year after T0) with the aim of an individualized positioner. In this study, all the implants with inclination equal to or greater than $10^{\circ}$ were subdivided into three groups, and the implants with inclination between 0 and 10 were used as control sample.

Results: In group I, there is a statistically significant difference in at least one of the two sides (distal one) between T0 and T1. In groups II and III, there is a statistically significant difference in the degree of bone resorption on the mesial side with respect to distal side. In group IV, a statistically significant difference on both sides was evidenced. The implant survival at a distance of 1 year was equal to $100 \%$ of cases.

Conclusion: Surgeons must take into account the possibility that an increase in inclination of implants may lead to a more rapid resorption of bone mesially or distally.

Clinical significance: The direction of the prosthetic load transmitted to the fixture is a variable that could influence the degree of reabsorption of both mesial and distal bone structures according to both laboratory and clinical data.

Keywords: Bone resorption, Implant complications, Implants, Osseointegration, Tilted implants.

The Journal of Contemporary Dental Practice (2020): 10.5005/jp-journals-10024-2799
\end{abstract}

\section{INTRODUCTION}

In dentistry, where new technologies are constantly applied leading to a continuous improvement in therapies, ${ }^{1-9}$ osseointegrated implants, that represent one of the most evolving branch, are today widely used for partial or total rehabilitation of edentulous spaces. ${ }^{10}$

To obtain an optimal anchorage of the implant within the bone plate, the surgeon should take into account the anatomy of the maxillary and mandibular regions: in the maxillary arch, the presence of the maxillary sinuses can sometimes make implant placement difficult in the posterior sectors; in the mandibular arch, the presence of the alveolar neurovascular bundle can make distal implant placement impossible if the level of bone atrophy is high.

To overcome these problems, some procedures of regenerative dentistry have been described: maxillary sinus lift represents a solution that allow implant positioning in the maxillary arch; even in the mandibular arch, it is possible to carry out regenerative procedures, through the use of bone grafts. These procedures are widely used and described in scientific literature, but it is unavoidable that they lead to increase morbidity for the patients.

As an alternative, it is possible to place inclined implants, to obtain sufficient primary stability.

Oblique implants have been adopted to increase retention in residual bone and to avoid anatomical site at risk. Malò et al. and Daverio et al. in their papers pointed out the use of non-bicortical implants. ${ }^{11,12}$

In case of excessive pressure, bone resorption has been evidenced over time: fibroblasts appear, and fibrous connective tissue is formed to replace bony tissue. This kind of process is probably the reason that explains cases of peri-implantitis due to masticatory overloading. ${ }^{13}$

\footnotetext{
${ }^{1-4}$ Department of Surgery and Medicine, University of Milano-Bicocca, Monza, Italy
}

Corresponding Author: Mirabelli Luca, Department of Surgery and Medicine, University of Milano-Bicocca, Monza, Italy, Phone: +39 3486419399, e-mail: dott.lucamirabelli@gmail.com

How to cite this article: Maddalone $M$, Mirabelli $L$, Breschigliaro $S$, et al. Radiographic Evaluation of the Bone Remodeling on Tilted Osseointegrated Implants: Follow-up at 1 Year. J Contemp Dent Pract 2020;21(4):463-470.

Source of support: Nil

Conflict of interest: None

Other possible complications include the possibility of irritation of the alveolar nerve that lead to problems of sensitivity and the possibility of penetration of the implant in the maxillary sinus.

Oblique bicortical or non-bicortical screw fractures are rare and regarded as fatigue fractures occurring time after insertion in the presence of sclerotic bone. ${ }^{14}$ Oblique implants do not have other noticeable complications.

\section{Purpose of Research}

The aim of this study was to evaluate the degree of vertical periimplant resorption that occurred around implants inserted both in the mandible and in the maxilla, with an inclination of more than $10^{\circ}$. The negative results were compared with those obtained by calculating the vertical bone resorption around straight implants, inclined less than $10^{\circ}$. In the same cohort of patients, we also evaluated implant survival at a distance of at least 1 year from the insertion of the fixtures.

() The Author(s). 2020 Open Access This article is distributed under the terms of the Creative Commons Attribution 4.0 International License (https://creativecommons. org/licenses/by-nc/4.0/), which permits unrestricted use, distribution, and non-commercial reproduction in any medium, provided you give appropriate credit to the original author(s) and the source, provide a link to the Creative Commons license, and indicate if changes were made. The Creative Commons Public Domain Dedication waiver (http://creativecommons.org/publicdomain/zero/1.0/) applies to the data made available in this article, unless otherwise stated. 


\section{Materials and Methods}

For the realization of the study, a sample consisting of 47 patients for a total of 115 implants was selected.

\section{Inclusion Criteria}

- Partial or total edentulous patients;

\section{Exclusion Criteria}

- Presence of general medical conditions that interfered with surgery

- Severe atrophy resulting from cancer surgery or trauma that make impossible to proceed with implant placement

- Smoking patients

- Patients undergoing radiotherapy for less than 2 years

- Insufficient accessibility to the oral cavity

Before the insertion of the fixtures, an orthopantomography of the dental arches was done using a radiographic panoramic Orthophos XG 3D Sirona with the aim of an individualized positioner to be able to perform a new examination in the same conditions at the follow-up of at least 1 year (before the insertion of the definitive prosthesis).

All patients were given antibiotic-based therapy: amoxicillin added to clavulanic acid ( $1 \mathrm{~g} / \mathrm{cp}$ ) to be taken every 8 hours starting from the second day before the surgery and continued for a total of 10 days to prevent postoperative infections.

Furthermore, a single dose of betamethasone sodium phosphate (4 mg i.m.) was administered after surgery to all patients.

Surgery has been performed under local anesthesia (mepivacaine and vasoconstrictor 1:100,000); a mucoperiosteal flap was made to access the bone structure below, and through the use of dedicated drills, we proceeded to implant insertion with variable inclination.

All patients received "Neoss" implants ProActive-Straight ${ }^{\circledR}$ with a length of $13 \mathrm{~mm}$ and a diameter of $4 \mathrm{~mm}$.

The entire preparation of the implant site was performed under abundant irrigation with refrigerated saline solution and with an intermittent milling technique. This avoids the overheating of bone and creates a pump effect for an efficient removal of milling debris.

After careful preparation of the surgical site, the implant was inserted. The mechanical installation of the implant was performed at low speed, about $20 \mathrm{rpm}$, with a controlled torque, not exceeding $45 \mathrm{~N} \mathrm{~cm}$. If it was necessary to use a higher torque the last implant portion was inserted manually.

Once implant placement was completed, the surgical suture was made with the use of a silk suture of 4-0. Implants tested were loaded between 8 weeks and 16 weeks after insertion.

In some patients, it was necessary to increase the bone volume by maxillary sinus augmentation. When a bone augmentation procedure was done, the time before loading was extended to 24 weeks.

\section{Collection of Radiographic Data}

Radiographic examination was performed in two postoperative timings:

- T0 corresponding to an initial radiographic check, before prosthetic loading of the implant;

- T1 corresponding to a later postoperative control in a period ranging from 12 months to 24 months after prosthetic loading.
Digital panoramic radiographs have been acquired with a specific image analysis software (Sidexis by Sirona ${ }^{\circledR}$ ); to standardize the analyses for each group of patients, vertical measurements of peri-implant bone were all performed using as reference the occlusal plane meaning tracing a line that touched the cusps of the dental elements in the same arch where the inclined implants were located. The analyzed images were all performed with the same orthopantomograph, and using a dedicated software ensured that the patient was positioned with the Frankfurt plane parallel to the floor using the individualized positioner to minimize image distortions and avoid dimensional alterations of the generated images.

The insertion implant angle was then calculated by measuring the angle between the greater axis of the implant and the perpendicular to the occlusal plane.

To minimize the acquisition of distorted measurements, each measurement was detected along an axis parallel to the greater axis of the implant, tangentially to the mesial and distal margins of the implant at T0, before the prosthetic loading.

The measurements thus obtained were then compared with those obtained at T1, with a minimum time of 12 months after implant loading.

In this study, all the implants with inclination equal to or greater than $10^{\circ}$ were took into consideration; implants with inclination between 0 and 10 were used as control sample.

The following groups have been identified:

- Implants not inclined: inclination between $0^{\circ}$ and $10^{\circ}$;

- Implants with inclination between $10^{\circ}$ and $20^{\circ}$;

- Implants with inclination between $20^{\circ}$ and $30^{\circ}$;

- Implants with inclination greater than or equal to $30^{\circ}$.

The data thus obtained were classified in Table 1.

\section{Statistical Analysis}

The data collected were analyzed using the software Prism 6.0 (GraphPad Software, Inc., La Jolla, CA, USA). The obtained evaluations have been divided into four different groups of study: Group I: Inclination between $0^{\circ}$ and $10^{\circ}$ (Tables 2 to 4)

Group II: Inclination included between $10^{\circ}$ and $20^{\circ}$ (Tables 5 to 7 )

Group III: Inclination included between $20^{\circ}$ and $30^{\circ}$ (Tables 8 to 10)

Group IV: Inclination above $30^{\circ}$ (Tables 11 to 13 )

\section{Results}

As shown in the previous tables on the different groups of patients, the "Column statistic of paired $t$ test" allowed us to compare mesial bone level at T0 with the mesial bone level at T1 and, in the same way, the distal bone level in both times.

In all four groups as the normal test was passed, we could perform the "Paired $t$ test of paired $t$ test" that allowed us to obtain a $p$ value and evaluate if there was a statistically significant difference in the degree of bone resorption on implants with increased inclination, compared with those with lower inclination.

In group I, we can conclude that there is a statistically significant difference in at least one of the two sides (distal one) between T0 and T1. In groups II and III, there is a statistically significant difference in the degree of bone resorption on the mesial side with respect to distal side.

In group IV, taking into account the lack of the sample, we can state that there is a statistically significant difference on both sides. 


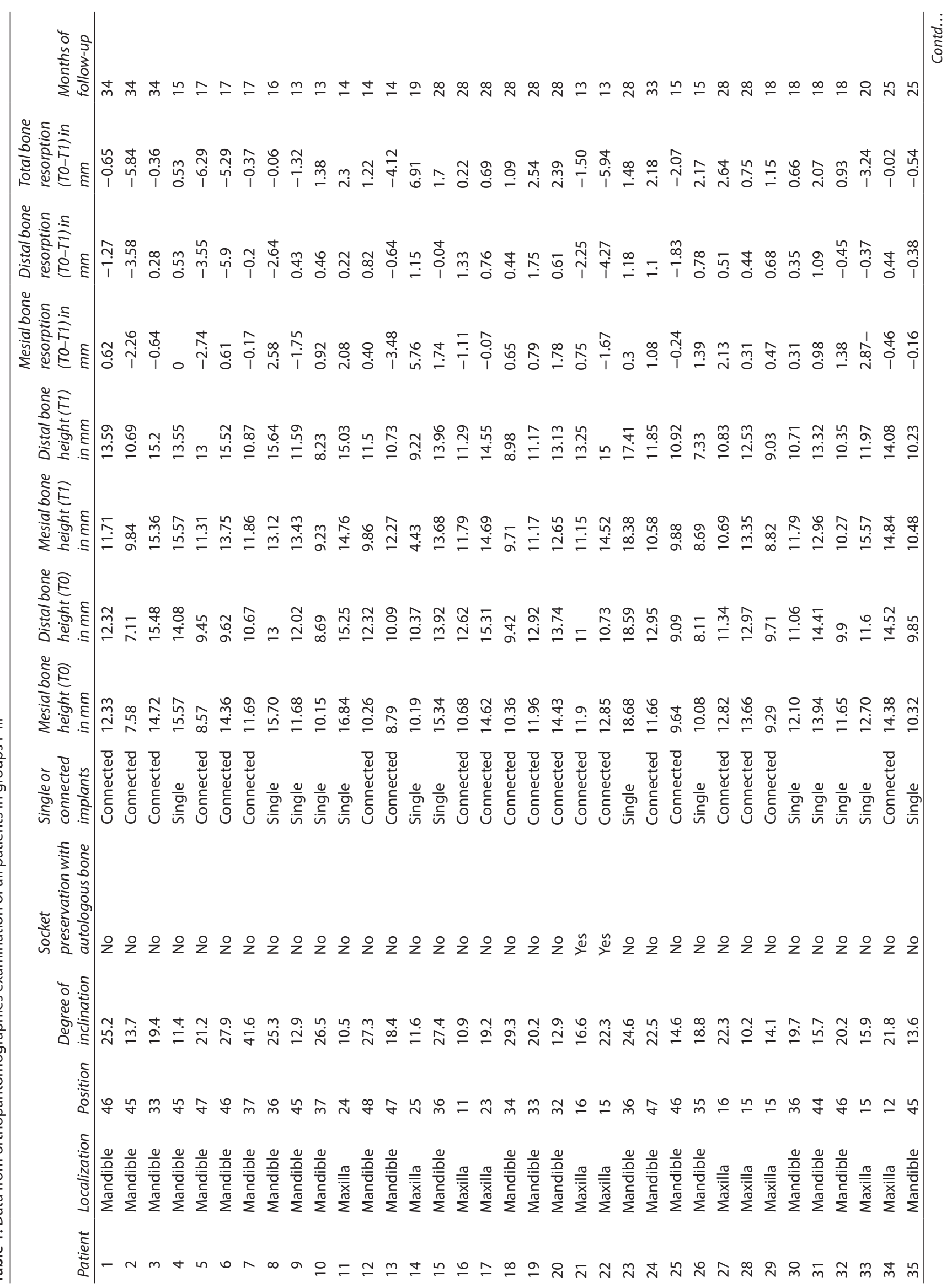




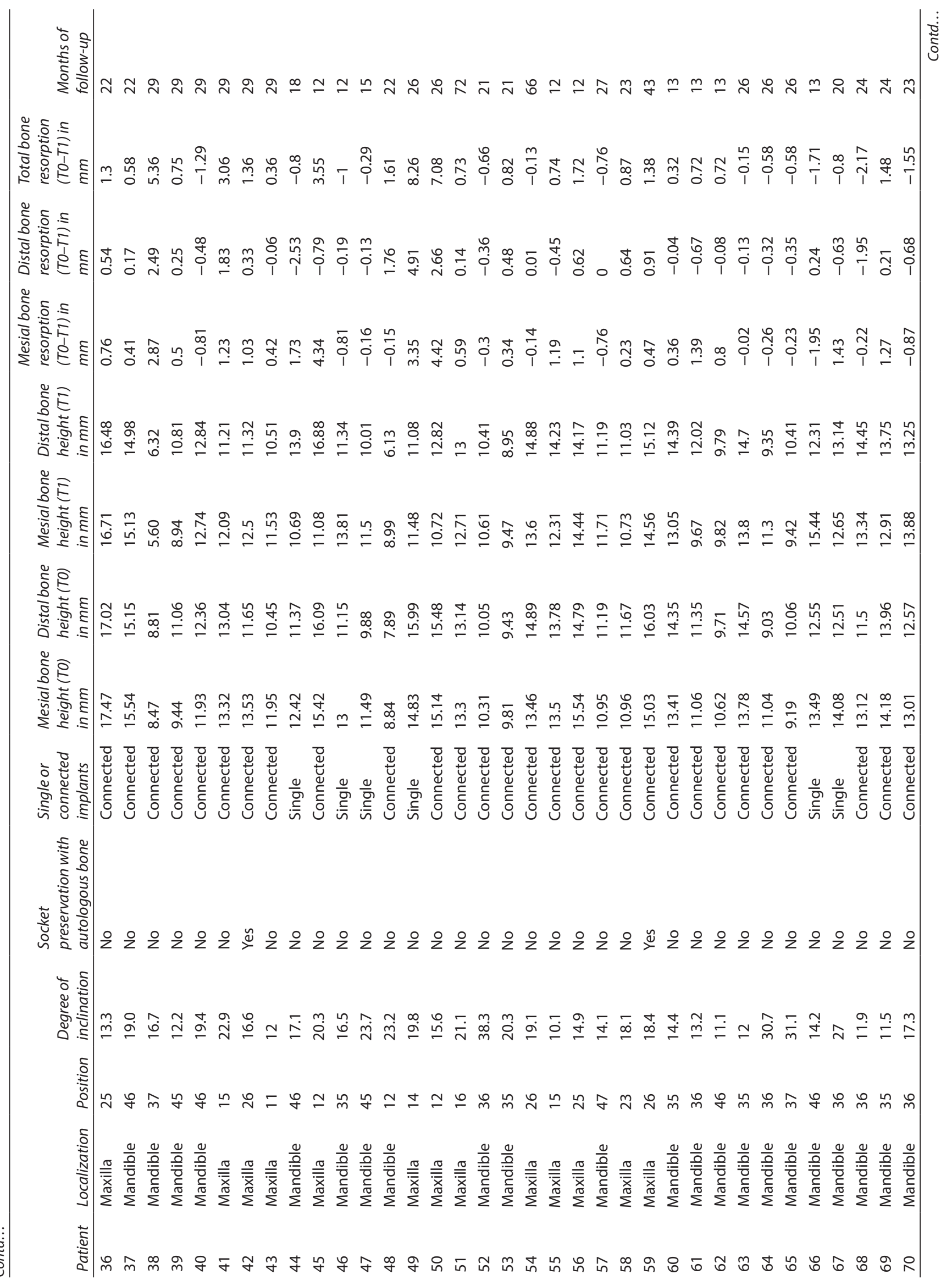




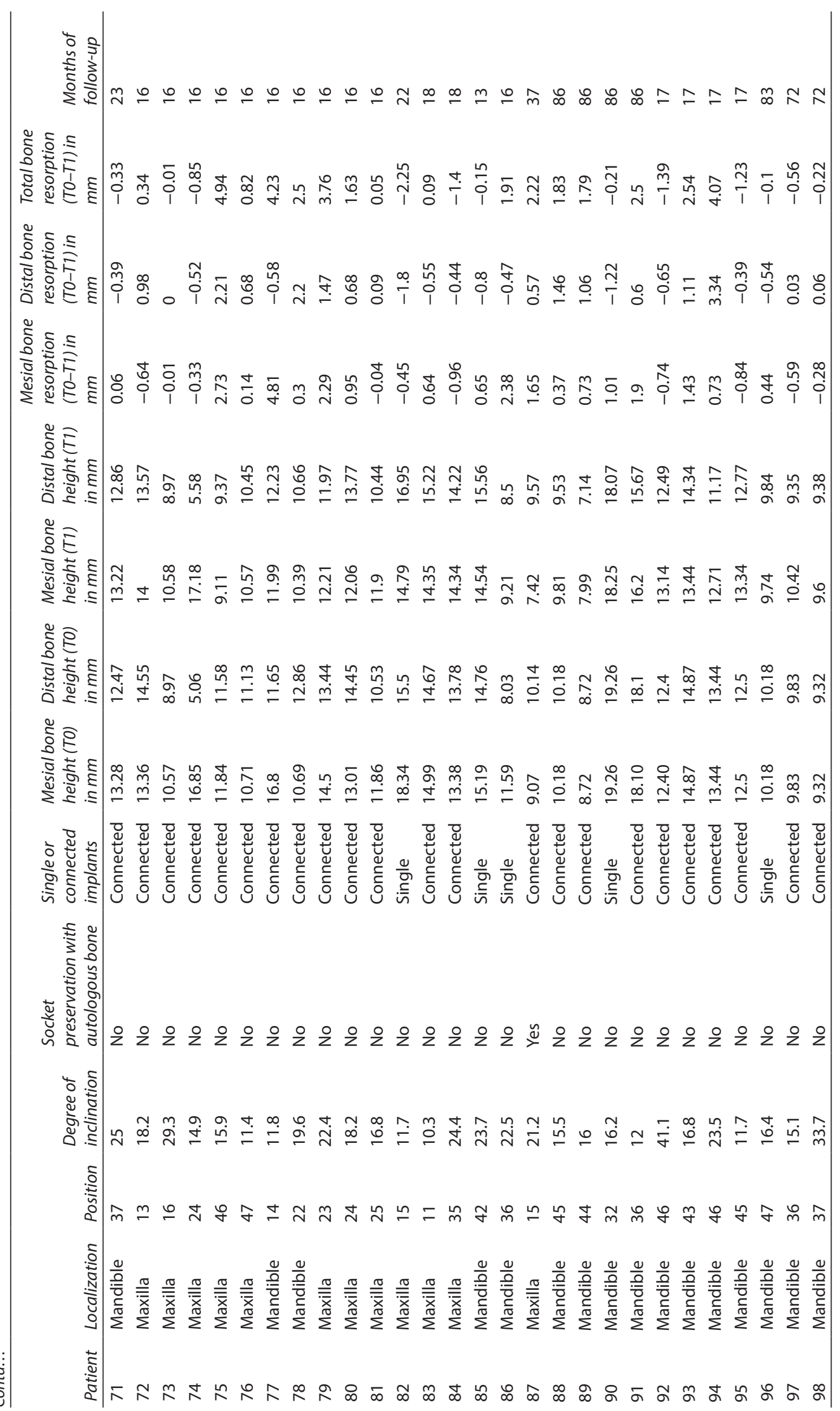


Table 2: D'Agostino-Pearson omnibus normality test

\begin{tabular}{lllll}
\hline & $\begin{array}{l}\text { Bone length } \\
\text { mesial T0 }\end{array}$ & $\begin{array}{l}\text { Bone length } \\
\text { mesial T1 }\end{array}$ & $\begin{array}{l}\text { Bone length } \\
\text { distal T0 }\end{array}$ & $\begin{array}{l}\text { Bonelength } \\
\text { distal T1 }\end{array}$ \\
\hline K2 & 1.612 & 2.241 & 0.1794 & 0.5975 \\
$p$ value & 0.4467 & 0.3261 & 0.9142 & 0.7418 \\
$\begin{array}{l}\text { Passed } \\
\text { normality test } \\
(\alpha=0.05) ?\end{array}$ & Yes & Yes & Yes & Yes \\
\hline
\end{tabular}

Table 3: Paired $t$ test bone length total mesial T0 vs bone length total mesial T1

\begin{tabular}{ll}
\hline$p$ value & 0.4529 \\
Significantly different $(p<0.05)$ & No \\
One- or two-tailed $p$ value & Two-tailed \\
$t, d f$ & $t=0.7694, d f=16$ \\
Numbers of pairs & 17 \\
\hline
\end{tabular}

Table 4: Paired $t$ test bone length total distal T0 vs bone length total distal T1

\begin{tabular}{ll}
\hline$p$ value & 0.0411 \\
Significantly different $(p<0.05)$ & Yes \\
One- or two-tailed $p$ value & Two-tailed \\
$t, d f$ & $t=2,222, d f=16$ \\
Numbers of pairs & 17 \\
\hline
\end{tabular}

Table 5: D'Agostino-Pearson omnibus normality test

\begin{tabular}{lllll}
\hline & $\begin{array}{l}\text { Bone length } \\
\text { mesial T0 }\end{array}$ & $\begin{array}{l}\text { Bone length } \\
\text { mesial T1 }\end{array}$ & $\begin{array}{l}\text { Bone length } \\
\text { distal T0 }\end{array}$ & $\begin{array}{l}\text { Bone length } \\
\text { distal T1 }\end{array}$ \\
\hline K2 & 1.298 & 1.671 & 1.271 & 0.9531 \\
$p$ value & 0.5225 & 0.4336 & 0.5296 & 0.6209 \\
$\begin{array}{l}\text { Passed } \\
\text { normality test } \\
(\alpha=0.05) ?\end{array}$ & Yes & Yes & Yes & Yes \\
\hline
\end{tabular}

Table 6: Paired $t$ test bone length total mesial T0 vs bone length total mesial T1

\begin{tabular}{ll}
\hline$p$ value & 0.0194 \\
Significantly different $(p<0.05)$ & Yes \\
One- or two-tailed $p$ value & Two-tailed \\
$t, d f$ & $t=2.401, d f=61$ \\
Numbers of pairs & 62 \\
\hline
\end{tabular}

Table 7: Paired $t$ test bone length total distal T0 vs bone length total distal T1

\begin{tabular}{ll}
\hline$p$ value & 0.2471 \\
Significantly different $(p<0.05)$ & No \\
One- or two-tailed $p$ value & Two-tailed \\
$t, d f$ & $t=1.169, d f=61$ \\
Numbers of pairs & 17 \\
\hline
\end{tabular}

Implant survival at a distance of 1 year was equal to $100 \%$ of cases. No neurological complications occurred at the site of the implants; in only eight patients, we noticed the presence of a copious bleeding, which caused the formation of a hematoma remained evident for 10 days (probably because five of these eight patients regularly took antiplatelet therapy).
Table 8: D'Agostino-Pearson omnibus normality test

\begin{tabular}{lllll}
\hline & $\begin{array}{l}\text { Bonelength } \\
\text { mesial T0 }\end{array}$ & $\begin{array}{l}\text { Bone length } \\
\text { mesial T1 }\end{array}$ & $\begin{array}{l}\text { Bonelength } \\
\text { distal T0 }\end{array}$ & $\begin{array}{l}\text { Bonelength } \\
\text { distal T1 }\end{array}$ \\
\hline K2 & 0.5960 & 3.590 & 1.086 & 0.5884 \\
$p$ value & 0.7423 & 0.1661 & 0.5810 & 0.7451 \\
$\begin{array}{l}\text { Passed } \\
\text { normality test } \\
(\alpha=0.05) ?\end{array}$ & Yes & Yes & Yes & Yes \\
\hline
\end{tabular}

Table 9: Paired $t$ test bone length total mesial T0 vs bone length total mesial T1

\begin{tabular}{ll}
\hline$p$ value & 0.0042 \\
Significantly different $(p<0.05)$ & Yes \\
One- or two-tailed $p$ value & Two-tailed \\
$t, d f$ & $t=3,104, d f=29$ \\
Numbers of pairs & 30 \\
\hline
\end{tabular}

Table 10: Paired $t$ test bone length total distal T0 vs bone length total distal T1

\begin{tabular}{ll}
\hline$p$ value & 0.6696 \\
Significantly different $(p<0.05)$ & No \\
One- or two-tailed $p$ value & Two-tailed \\
$t, d f$ & $t=0,4310, d f=29$ \\
Numbers of pairs & 30 \\
\hline
\end{tabular}

Table 11: Kolmogorov-Smirnov test with Dallal-Wilkinson-Lillie for $p$ value

\begin{tabular}{lcccc}
\hline & $\begin{array}{l}\text { Bonelength } \\
\text { mesial T0 }\end{array}$ & $\begin{array}{l}\text { Bone length } \\
\text { mesial T1 }\end{array}$ & $\begin{array}{l}\text { Bone length } \\
\text { distal T0 }\end{array}$ & $\begin{array}{l}\text { Bone length } \\
\text { distal T1 }\end{array}$ \\
\hline KS & 0.1837 & 0.1700 & 0.2156 & 0.2029 \\
$p$ value & $>0.1000$ & $>0.1000$ & $>0.1000$ & $>0.1000$ \\
$\begin{array}{l}\text { Passed } \\
\text { normality test } \\
(\alpha=0.05) ?\end{array}$ & Yes & Yes & Yes & Yes \\
\hline
\end{tabular}

Table 12: Paired $t$ test bone length total mesial T0 vs bone length total mesial T1

\begin{tabular}{ll}
\hline$p$ value & 0.0111 \\
Significantly different $(p<0.05)$ & Yes \\
One- or two-tailed $p$ value & Two-tailed \\
$t, d f$ & $t=3.926, d f=5$ \\
Numbers of pairs & 6 \\
\hline
\end{tabular}

\section{Discussion}

Modern implantology allows to rehabilitate even extreme situations that a few decades ago were impossible to treat with predictability, improving patient's quality of life.

Necessary condition to obtain success in implantology is sufficient bone structure to guarantee primary and secondary stability of the implants.

Furthermore, with the use of regenerative techniques, patients had the possibility to rehabilitate their dentition using fixed implantsupported prostheses.

In recent years, a technique called "All on four" introduced the possibility to rehabilitate completely edentulous jaws thanks to the 
Table 13: Paired $t$ test bone length total distal T0 vs bone length total distal T1

\begin{tabular}{ll}
\hline$p$ value & 0.0238 \\
Significantly different $(p<0.05)$ & Yes \\
One- or two-tailed $p$ value & Two-tailed \\
$t, d f$ & $t=3.206, d f=5$ \\
Numbers of pairs & 6 \\
\hline
\end{tabular}

insertion of four implants in which the two are distally positioned with an inclination of more than $30^{\circ} .11$

This new technique stimulated numerous studies on the positioning and clinical success of inclined implants.

The question whether tilted implants are more at risk of failure than axially placed implants is receiving increasing attention in the last years, and a periodic review of the different concepts is necessary to refine techniques and eliminate unnecessary procedures.

We tried to examine whether increased inclination could create bone resorption higher than what was highlighted around implants positioned with inclinations less than $30^{\circ}$.

Chrcanovic et al. suggested that the differences in angulations of dental implants might not affect implant survival neither reduce marginal bone levels. ${ }^{15}$

Del Fabbro et al. found no significant difference in failure rate between tilted and upright implants, both maxillary and mandibular implants. No implant-supported prosthesis failure was reported. Limited bone loss around the fixtures was reported with no difference between upright and tilted implants. In three studies examined, based on the questionnaires, a full satisfaction for function, phonetics, and esthetics was reported. For these reasons, they stated that the use of tilted implants to support immediately loaded fixed prostheses for the rehabilitation of edentulous arches can be considered a predictable technique, with an excellent prognosis in the short-medium term even if they recommended randomized long-term trials to better state the efficacy of this surgical approach. ${ }^{16}$

Menini et al. in the same year stated that the outcomes of upright and tilted implants supporting full-arch fixed dentures for the immediate rehabilitation of edentulous maxillae, after at least 1 year of function in an electronic search of 1,069 articles, showed no significant difference in failure ( $p$ value $=0.52$ ).

A nonsignificant difference between tilted and upright implants was found with regard to bone loss, and tilted implants demonstrated a favorable short-term prognosis in full-arch immediate loading rehabilitations of the maxillae. Also, these authors observed that randomized long-term trials are needed to better explain long-term success of tilted vs upright-positioned implants. ${ }^{17}$

Similarly, Francetti et al. found that no implant failures were recorded to date, leading to a cumulative implant survival and prosthesis success rate of $100 \%$. Plaque level and bleeding scores showed a progressive decrease over time during the study, with simultaneous increase of satisfaction for both esthetic and function in the majority of patients. No significant difference in marginal bone loss was found between tilted and axial implants at 1-year follow-up. The authors concluded that tilted implants result a viable treatment modality for the mandible. ${ }^{18}$

Bellini et al. on the other way, with an experimental study, stated that tilted configurations of osseointegrated implants showed a lower absolute value of compressive stress compared with nontilted implants, indicating a possible biomechanical advantage in reducing stresses at the bone-implant interface. ${ }^{19}$

Our research, similarly and from a clinical perspective, has shown that there is a statistically significant difference in the degree of bone resorption for implants with inclination of more than $30^{\circ}$ as assessed from the analysis of the previous tables, even if it is still necessary to take into account the lack of the sample analyzed in the most inclined implants.

\section{ConCLusion}

Fixed rehabilitation technique using osseointegrated implants is a well-established practice.

As already described, some anatomical conditions make necessary to insert inclined implants in areas where bone or peripheral nerve structures do not allow the insertion of an upright implant with a suitable size to guarantee both primary and secondary stabilities. During the planning phase, surgeons must take into account the possibility that this increase in inclination may lead to a more rapid resorption of bone mesially or distally to the implant.

As previously described, the extent of resorption is correlated to the degree of inclination during implant insertion.

\section{Clinical Significances}

Previous articles many times found that no significant influence in overall implant survival exists, but an important factor to take into account is the direction of the prosthetic load transmitted to the fixture as this variable could influence the degree of resorption of both mesial and distal bone structure according to both laboratory and clinical data.

\section{References}

1. Venino PM, Citterio CL, Pellegatta A, et al. A micro-computed tomography evaluation of the shaping ability of two nickel-titanium instruments, Hyflex EDM and ProTaper next. J Endod 2017;43(4): 628-632. DOI: 10.1016/j.joen.2016.11.022.

2. Maddalone M, Ferrari M, Stanizzi A, et al. Use of miniscrew implants in orthodontic distal movement [Utilizzo delle miniviti nelle meccaniche ortodontiche di distalizzazione]. Dental Cadmos 2010;78(8): 97-105.

3. Maddalone M, Ferrari M, Barrila' $S$, et al. Intrusive mechanics in orthodontics with the use of TAD's [Utilizzo delle miniviti nelle meccaniche ortodontiche di intrusione]. Dent Cadmos 2010;78(7): 97-106.

4. Maddalone M. The association between the psychological status and the severity of facial deformity in orthognathic patients. Angle Orthodontist 2012;82(3):396-402. DOI: 10.2319/060211-363.1.

5. Citterio F, Pellegatta A, Citterio $C L$, et al. Analysis of the apical constriction using micro-computed tomography and anatomical sections. G Ital Endod 2014;28(1):41-45. DOI: 10.1016/ j.gien.2014.05.001.

6. Galluzzi F, Pignataro L, Maddalone M, et al. Recurrences of surgery for antrochoanal polyps in children: A systematic review. Int J Pediatr Otorhinolaryngol 2018;106:26-30.

7. Maddalone $M$, Gagliani $M$, Citterio $C L$, et al. Prevalence of vertical root fractures in teeth planned for apical surgery. A retrospective cohort study. Int Endod J 2018;51(9):969-974.

8. Maddalone M, Mirabelli L, Venino PM, et al. Long-term stability of autologous bone graft of intraoral origin after lateral sinus floor elevation with simultaneous implant placement. Clin Implant Dent Relat Res 2018;20(5):713-721. DOI: 10.1111/cid.12649. 
9. Porcaro G, Busa A, Bianco E, et al. Use of a partial-thickness flap for guided bone regeneration in the upper jaw. J Contemp Dent Pract 2017;18(12):1117-1121. DOI: 10.5005/jp-journals-100242186.

10. Cavalli N, Corbella S, Taschieri S, et al. Prevalence of periimplant Mucositis and peri-implantitis in patients treated with a combination of axial and tilted implants supporting a complete fixed denture. Sci World J 2015;2015:874842. DOI: 10.1155/2015/ 874842.

11. Malò $P$, de Araújo Nobre $M$, Lopes $A$. The use of computer-guided flapless implant surgery and 4 implants placed in immediate function to support a fixed denture: preliminary results after a mean follow-up period of 13 months. J Prosthet Dent 2007;97(6 Suppl):26-34. DOI: 10.1016/S0022-3913(07)60005-5.

12. Daverio L, Franchini I, Capelli M, et al. Prospective longitudinal study in the rehabilitation of the edentulous maxilla with oblique distal implants. Oral Implantol Milan 2007;3:18-25.

13. Emanuelli M, Di Lollo S, Paglierani M, et al. Perimplantary histopathological study with linkow blades. Odontostomatol Implantoprothesis 1989;7:10-21.
14. Lindquist LW, Rockler B, Carlsson GE. Prognosis for osseointegrated implants. J Prosthet Dent 1988;59(1):59-63. DOI: 10.1016/00223913(88)90109-6.

15. Chrcanovic BR, Albrektsson T, Wennerberg A. Tilted versus axially placed dental implants: a meta-analysis. J Dent 2015:43(2):149-170. DOI: 10.1016/j.jdent.2014.09.002.

16. Del Fabbro M, Bellini CM, Romeo $D$, et al. Tilted implants for the rehabilitation of edentulous jaws: a systematic review. Clin Implant Dent Relat Res 2012;14(4):612-621. DOI: 10.1111/j.17088208.2010.00288.x.

17. Menini M, Signori A, Tealdo T, et al. Tilted implants in the immediate loading rehabilitation of the maxilla: a systematic review. J Dent Res 2012;91(9):821-827. DOI: 10.1177/0022034512455802.

18. Francetti L, Agliardi E, Testori T, et al. Immediate rehabilitation of the mandible with fixed full prosthesis supported by axial and tilted implants: interim results of a single color prospective study. Clin Implant Dent Relat Res 2008;10(4):255-263. DOI: 10.1111/j.17088208.2008.00090.x.

19. Bellini $C M$, Romeo D, Galbusera F, et al. A finite elements analysis of tilted versus non tilted implant configurations in the edentulous maxilla. Int J Prosthodont 2009;22(2):155-157. 